\title{
Multifunctional Integrated Photonic Switches for Nanosecond Packet-Switched Wavelength Conversion
}

\author{
Onur Fidaner, Hilmi Volkan Demir ${ }^{1}$, Vijit A. Sabnis ${ }^{2}$, James S. Harris, Jr., and David A. B. Miller \\ Edward L. Ginzton Laboratory, Stanford University, Stanford, California 94305 USA \\ ${ }^{1}$ also with Bilkent University, Ankara, Turkey 06800 \\ ${ }^{2}$ currently with Translucent, Inc., Palo Alto, CA 94303 USA \\ ofidaner@stanford.edu \\ Jun-Fei Zheng \\ Strategic Technology, Intel Corporation, Santa Clara, California 95052 USA
}

\begin{abstract}
We report multifunctional integrated photonic switches that provide optical wavelength conversion across the $\mathrm{C}$-band at $3.5 \mathrm{~Gb} / \mathrm{s}$ that is electrically packet-switched within a reconfiguration time of $<2.5 \mathrm{~ns}$. These switches also provide optical packet-switching in $<300 \mathrm{ps}$. (C) 2005 Optical Society of America

OCIS codes: (250.3140) Integrated optoelectronic circuits; (060.2330) Fiber optics communications
\end{abstract}

\section{Introduction}

Multifunctionality of optical network nodes is becoming increasingly a critical aspect for the development of future optical networks. To accommodate the rapid growth in the network traffic and optimally compensate for fluctuations in the network capacity usage, the network nodes should be capable of utilizing as many of the available physical domains as possible, i.e., wavelength, time, space, and code [1]. For that, it is desirable not only to map one channel in one of the domains to another channel in the same domain (such as wavelength conversion), but also to map one domain to another (such as wavelength-space mapping) within a reasonable reconfiguration time. Various devices have been proposed by a number of research groups to realize one or more of these network functions, such as regeneration [2], fast switching [3], wavelength conversion [4], WDM to TDM conversion [4], and packet switching [5]. In this work, we demonstrate multifunctional integrated photonic switches that simultaneously perform a number of such network functions, including unconstrained C-band wavelength conversion and packet switching with either electrical or optical control signals. The switch architecture is further scalable in two dimensions, which makes it possible to fabricate crossbar switches to offer space switching as well [6].

\section{Device Concept and Switch Operation}

In our device, we intimately integrate a waveguide electroabsorption modulator (EAM) with a surface-illuminated photodiode (PD) such that the PD directly drives the EAM [6-8]. Fig. 1 illustrates the integrated photonic switch. The PD is a surface-normal $p$ - $i-n$ diode comprising a $1.25 \mu \mathrm{m}$ thick InGaAs absorber region that strongly absorbs over the 1.3-1.6 $\mu \mathrm{m}$ telecommunication wavelength band. The EAM, on the other hand, is a waveguide $p-i-n$ diode with an InGaAsP multiple quantum well (MQW) structure that is optimized for large optical modulation over the Cband. Using a surface-normal PD renders the switch insensitive to input signal polarization and allows for polarization resetting. The PD and the EAM are tightly integrated using a selective area regrowth technique [9]. Such an intimate integration, in which the overall chip size is confined to an area of $300 \mu \mathrm{m}$ by $300 \mu \mathrm{m}$, provides lumped-circuit operation of the integrated parts. As a result, the 3-dB switching bandwidth is predominantly determined by the internal device RC time constant. The DC bias is applied through a set of external bypass capacitors to confine the high-speed signals within the switch and to prevent loading from the DC biasing circuit.

In the switch, there are two optical inputs, one electrical input, and one optical output, as shown in Fig 1 . The first optical input is a surface-normal, optical data stream incident onto the PD at wavelength $\lambda_{1}$ and the other optical input is a continuous-wave (cw) beam at wavelength $\lambda_{2}$ coupled into the waveguide EAM. The electrical input is a biasing electrical signal across the EAM. The optical output is the beam exiting the EAM waveguide.

As illustrated in the simplified circuit diagrams in Fig. 2, there are two possible configurations in which the switch can be operated. In both cases, the PD is DC-biased such that it generates photocurrent whenever there is incident optical data at wavelength $\lambda_{1}$. The resulting photocurrent, in turn, causes an almost instantaneous voltage drop across the on-chip, pull-up resistor (R), and consequently across the EAM. In the first configuration shown in Fig 2(a), the EAM is electrically enabled when reverse-biased and disabled when slightly forward-biased by the 
enabling and disabling periods of a biasing electrical signal that comes from the central network management system (CNMS). With the enabling CNMS signal, the DC operating point of the EAM is set such that it absorbs the $\mathrm{cw}$ input at $\lambda_{2}$. The voltage change resulting from the presence of optical data on the PD changes the absorption properties of the EAM, allowing for transmission of the cw input at $\lambda_{2}$. Thus, in the presence of the enabling CNMS signal, the input at $\lambda_{1}$ is bit-by-bit transferred to the output channel at $\lambda_{2}$. However, if the disabling CNMS signal is present across the EAM, then the optical data transfer (wavelength conversion from $\lambda_{1}$ to $\lambda_{2}$ ) is completely blocked despite the optical input data stream present at the PD. Therefore, the electrical CNMS signal gates the optical switching and the optical wavelength conversion can be packet-switched electrically.

In the second configuration, as illustrated in Fig 2(b), the EAM is constantly reverse biased by a DC signal. This time, the optical data stream is coupled into the EAM instead of the PD. The data is only transmitted when an optical control beam is present on the PD. This optical control signal comes from the CNMS and it again gates the data channel.

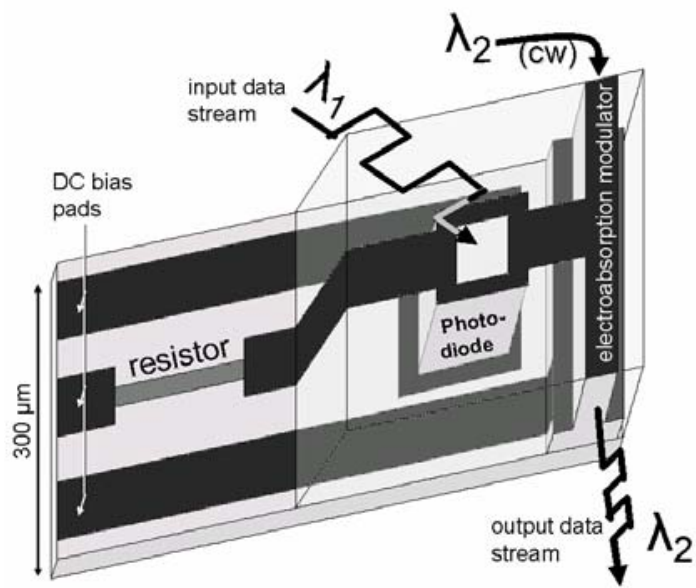

Fig.1. An illustration of the device integration. The PD and the EAM are separated by $50 \mu \mathrm{m}$.

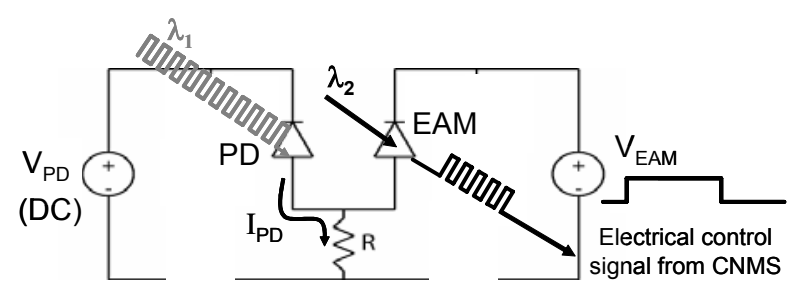

(a)

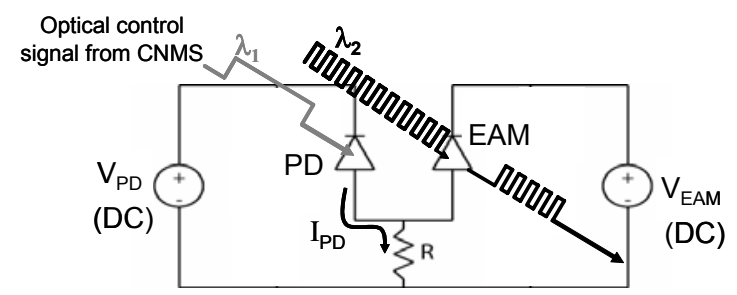

(b)

Fig.2. A simplified circuit diagram of the PD-EAM. The photocurrent, $I_{P D}$, from PD generates a voltage drop across the resistor $R$, reducing the voltage across EAM. This voltage reduction increases the optical transmission of the EAM provided it is properly reverse-biased. (a) Whenever an electrical signal (packet switching envelope) from the central network management system (CNMS) reverse-biases the EAM, the photocurrent-induced voltage change modulates the $\mathrm{cW}$ optical input of the EAM. Consequently, the input data at $\lambda_{1}$ is transmitted from the output channel at $\lambda_{2}$. (b) In this case, the EAM is reverse biased at all times. The optical data coupled into the EAM is transmitted only when the PD optically drives the EAM, i.e., when an optical control signal from CNMS is present on the PD.

\section{Experimental Results}

Unconstrained wavelength conversion and dual wavelength broadcasting have been demonstrated previously using these switches at $2.5 \mathrm{~Gb} / \mathrm{s}$ [7] and at $1.25 \mathrm{~Gb} / \mathrm{s}$ [8], respectively. Fig. 3 shows an exemplary wavelength conversion from the input wavelength $1551.7 \mathrm{~nm}$ to the output wavelength $1548.5 \mathrm{~nm}$ for an NRZ signal at $3.5 \mathrm{~Gb} / \mathrm{s}$, with $>10 \mathrm{~dB}$ extinction ratio for an input absorbed power of $\sim 10 \mathrm{~mW}$. These devices have a $3-\mathrm{dB}$ switching bandwidth of $2.2 \mathrm{GHz}$.

Fig. 4 shows packet switching using an electrical control input. In this case, the EAM is driven by an electrical signal at $62.5 \mathrm{MHz}$ with rise/fall times of $\sim 1 \mathrm{~ns}$. With data present on the PD at $\lambda_{1}=1551.7 \mathrm{~nm}$ and cw beam coupled into the EAM at $\lambda_{2}=1535.8 \mathrm{~nm}$, the EAM output is observed. The applied electrical signal to the EAM gates the 
optical switching in $<2.5 \mathrm{~ns}$ and, as a result, the optical wavelength conversion is electrically packet-switched in $<2.5 \mathrm{~ns}$.

Fig. 5 shows packet switching with an optical control input. Both the PD and the EAM are reverse biased in this case. A $3 \mathrm{~Gb} / \mathrm{s}$ pulse train is coupled into the EAM at $\lambda_{2}=1550 \mathrm{~nm}$ and the packet-switching optical signal, a square wave at $375 \mathrm{MHz}$ at wavelength $\lambda_{1}=1551.7$, is incident on the PD. The optical control signal at $\lambda_{1}$ gates the data at $\lambda_{2}$.

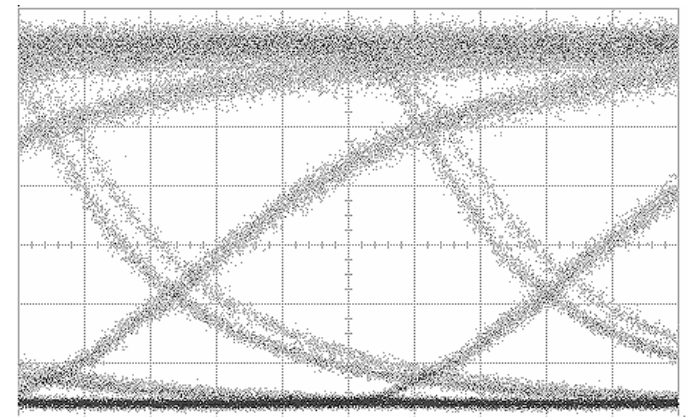

Fig.3. Eye diagram at $3.5 \mathrm{~Gb} / \mathrm{s}$ with $>10 \mathrm{~dB}$ extinction ratio

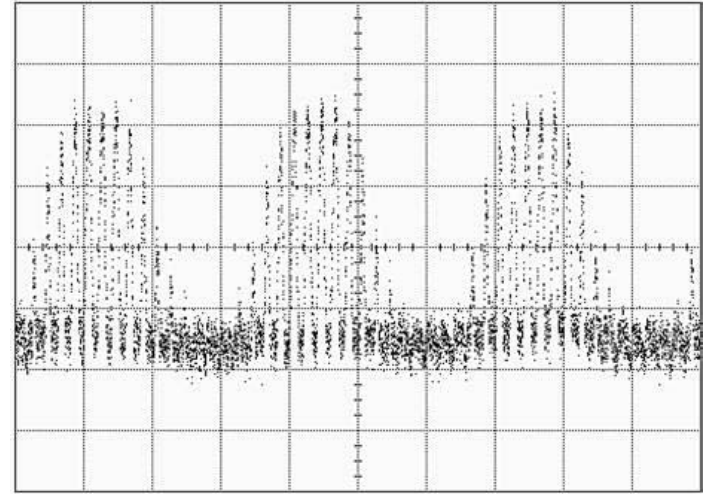

Fig. 4. Electrical packet switching of $1 \mathrm{~Gb} / \mathrm{s}$ wavelength conversion (from $1551.7 \mathrm{~nm}$ to $1535.8 \mathrm{~nm}$ ) at $62.5 \mathrm{MHz}$ within a reconfiguration time of $<2.5 \mathrm{~ns}$. ( $5 \mathrm{~ns} /$ square)

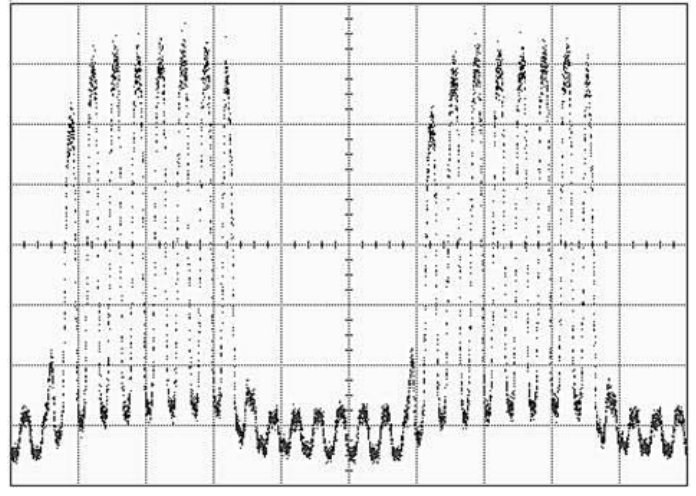

Fig. 5. Optical packet-switching of $3 \mathrm{~Gb} / \mathrm{s}$ optical pulse train (1550nm) with an optical CNMS signal $(1551.7 \mathrm{~nm})$ at $375 \mathrm{MHz}$ within a reconfiguration time of $<300 \mathrm{ps}$ ( $1 \mathrm{~ns} /$ square).

\section{Conclusion}

Integrated PD-EAMs make multifunctional photonic switches that exhibit polarization-insensitive, low-power, highspeed operation with high extinction ratios across a wide wavelength band (full C-band). They simultaneously provide multiple network functions on a single photonic chip. For example, they perform both optical wavelength conversion (at $3.5 \mathrm{~Gb} / \mathrm{s}$ ) and electrical packet switching (in $<2.5 \mathrm{~ns}$ ) on the same optical channel using such a single switch. Alternatively, they provide optical packet switching using an optical control signal (in $<300 \mathrm{ps}$ ). The architecture also allows for fabricating two-dimensional arrays of these switches on a single chip to implement crossbar switches for simultaneous wavelength, space and packet switching. Based on our experimental verification of the lumped circuit model at $3.5 \mathrm{~Gb} / \mathrm{s}$, we anticipate the switch can be further scaled to $10 \mathrm{~Gb} / \mathrm{s}$ operation by reducing the on-chip resistor value and internal device capacitances.

\section{References}

[1] S.J.B.Yoo, “Advanced Optical Components for Next Generation Photonic Networks,” Proc. SPIE 5246, 224-234 (2003)

[2] T. Otani et al., IEEE Photon. Tech. Lett., 12 (4), pp. 431-433, (2000)

[3] S. Kodama, T. Yoshimatsu, and H. Ito, Electron. Lett., 2004, vol. 40, No. 9

[4] M. Hayashi et al., J. Light. Tech., 20(2), pp. 236-242, (2002).

[5] C. Sato et al., OFC, paper MF53, Los Angeles, CA, 2004

[6] H. V. Demir et al., IEEE Photonics Technology Letters, 16(10), pp. 2305-2307 (2004).

[7] V. A. Sabnis et al., Applied Physics Letters, 84(4), pp. 469-471, (2004).

[8] H. V. Demir et al., OSA Optics Express, 12(2), pp. 310-316, (2004).

[9] V. A. Sabnis et al., OSA Conference on Integrated Photonics Research (IPR), pp. 12-14, (OSA Technical Digest, Optical Society of America, Washington, DC, 2003). Paper IMB3. 